\title{
Design and evaluation of a new exoskeleton for gait rehabilitation
}

\author{
Ionut Daniel Geonea and Daniela Tarnita \\ Faculty of Mechanics, University of Craiova, Craiova, 200512, Romania \\ Correspondence to: Daniela Tarnita (tarnita.daniela@gmail.com)
}

Received: 19 March 2017 - Revised: 1 September 2017 - Accepted: 8 September 2017 - Published: 17 October 2017

\begin{abstract}
This work addresses the design and numerical characterization of a new exoskeleton solution for human leg motion assistance and rehabilitation. The exoskeleton solution is anthropomorphic, simple, low cost and easy to adapt on the human subject. The design aspect concerns the exoskeleton mechatronic structure, achieved in SolidWorks virtual environment. Numerical simulation is performed in MSC.ADAMS simulation environment. Obtained results for the exoskeleton computed motion are compared with those obtained from experimental walking of healthy subject. The prototype feasibility is studied both for design and operation aspect.
\end{abstract}

\section{Introduction}

Nowadays, the importance of measuring and analyzing gait variability has increased and it is more and more recognized and used in biomechanics, in robotics, in rehabilitation and in clinical research field. Clinical gait analysis usually consists of measurement of gait parameters, kinematic analysis, kinetic measurement and electromyography. Spatial and temporal parameters of gait provide useful diagnostic and therapeutic information, if they are accurately measured (Begg et al., 1989). The most analyzed parameters of normal and pathological human gait are the following: travelled distances, velocity, gait phases, step length, joints angles, swing time, support time, ground reaction forces, forces and momentum in joints (Tarnita, 2016). In the medical field, the knowledge of gait characteristics, the monitoring and evaluating changes in human gait reveal important information about quantitative objective measurement of the different gait parameters, about the evolution and early diagnosis of different diseases and about physical therapy, involving rehabilitation, which helps to improve the walking function (Sutherland et al., 2002; Tao et al., 2012; Muro-de-la-Herran et al., 2014). The main goal of the rehabilitation therapy is to minimize functional deficits of disabled patients, this procedure requiring a repetitive motion performed by the patient. For that purpose, the modern rehabilitation techniques use mechanical systems (as robots or exoskeletons) to assist the lower limbs in their movements' rehabilitation. The main advantages of robotic rehabilitation are: reducing dependence on clinical staff, providing adequate rehabilitation movements and adjusting the level of treatment according to patient requirements, allowing control of joint movement, helping ensure controlled repetitive preparation at a reasonable cost (Banala et al., 2007).

An exoskeleton is defined as an active mechanical device that is essentially anthropomorphic, in nature is "worn" by an operator and it augments the performance of an able-bodied wearer (Dollar et al., 2008). This purpose is realized by providing a supplementary force to the legs (Viteckova et al., 2013). Due to the increased interest upon the development of rehabilitation procedures based on mechatronic and robotic technologies, in recent years, many review studies which evaluate the progress and future directions in rehabilitation, were published (Onose et al., 2013; Young et al., 2017; Chen et al., 2013; Lajeunesse et al., 2016 and Louie et al., 2016). Ones of the most popular exoskeletons are:

- BLEEX (Berkely Lower Extremity Exoskeleton), pointed as the first load carrying exoskeleton (Anama et al., 2012). BLEEX exoskeleton actuates the hip abduction/adduction, hip flexion/extension and knee flexion;

- HAL (Hybrid Assistive Limb), described as a wearable robot intended for multiple applications, from rehabilitation purposes to heavy works additional support, (Díaz et al., 2011; Askani et al., 2016); 
- LOPES exoskeleton combine an actuated pelvis segment with a leg exoskeleton, with three actuated joints: two for hip and one for knee joint (Veneman et al., 2007).

- LOKOMAT system consists into a treadmill and a powered exoskeleton. In order to use this rehabilitation system, the patients that are affected by spinal cord injuries are sustained by a body weight support (Hesse et al., 2003).

- REX exoskeleton by REX Bionics is a wearable exoskeleton capable to support the weight of the patient and is able to self-balance (Barbareschi et al., 2015).

Multi joint exoskeletons are described by Tingfang et al. (2015), and most of them are based on the principle of predefined gait trajectory control. This principle according to which the exoskeleton replicates the desired joint trajectory corresponding to an experimental data set acquired from a healthy person is implemented to exoskeletons like HAL, Rewalk and Mindwalker (Wang et al., 2015a).

Exoskeletons for industrial application are presented in de Looze et al. (2016), powered exoskeletons in post - stroke rehabilitation are studied in Louie et al. (2016) and for arm rehabilitation in Schorsch et al. (2014); Jarrassé et al. (2014). The aspect of control systems of active exoskeletons is studied in Anama et al. (2012), while aspects of exoskeleton design, path planning, modeling and simulation, experimental tests are studied by Carbone et al. (2007); Ashkani et al. (2016); Wang et al. (2015b); Ceccarelli et al. (2009).

Recently, numerous review articles presenting the state of the art concerning passive devices used for rehabilitation of lower limb are published. These types of device are represented by passive functional upper-limb orthoses (Rahman et al., 2006).

According to the rehabilitation principle (Díaz et al., 2011), the active rehabilitation devices can be grouped in five groups: (a) treadmill gait trainers; (b) foot-plate-based gait trainers; (c) mobile robotics based solutions; (d) stationary gait trainers; (e) ankle rehabilitation systems, as active foot orthoses.

Some studies (Onose et al., 2016) evaluate the progress and future directions in the field of robotic rehabilitation technologies, from the perspective of professionals, engineers and users, while the main improvements needed for the development and functional optimization of practical exoskeletons are highlighted in Lajeunesse et al. (2016).

Another research theme from this subject area is the development and evaluation of a new leg robotic exoskeleton, called $\mathrm{H} 2$, intended for gait rehabilitation of the stroke survivors (Bortole et al., 2015). In order to guide the development of lower limb exoskeletons, some studies (Kao et al., 2010) had, as purpose, the understanding of how humans adapt to powered assistance.
From studied literature it results that there are many devices and robotic systems for human gait rehabilitation. These systems are intended to patients affected by spinal cord injuries, muscular dystrophy, spinal muscular atrophy, cerebral palsy and stroke. These disorders produce muscular weakness and this is the reason for the development of locomotion assistance robotic devices and necessity of gait rehabilitation (Rahman et al., 2006). A category of exoskeletons is intended to a single joint motion assistance (Ceccarelli et al., 2016), and other category assists the motion of two or more leg joints. Exoskeletons in this category use actuators for joints, command and control architectures, and are costly solutions that are difficult to deploy on a large scale (Geonea et al., 2013). As a conclusion these existing solutions are not accessible to disabled persons, although they assure proper requirements for rehabilitation.

The low-cost solution does not perform rehabilitation movements for all of the leg joints because they generally provide active rehabilitation movement only for the hip and knee joints. Other devices are used as passive solutions to provide rehabilitation movements that correspond to a specific rehabilitation therapy designed to recover walking for a particular joint such as the hip, knee or ankle. Several devices solutions are designed to reproduce human bipedal locomotion, but generally few of them provide approximately anthropomorphic movements (Geonea et al., 2015).

This research proposes a new leg exoskeleton design that provides support for the movement of the hip and knee joints. An experimental human walking test is used to obtain reference movement laws for the exoskeleton's joints during walking. Then, a dynamic study of the walking exoskeleton attached to a human virtual mannequin is performed. The dynamic virtual model is designed using SolidWorks, and numerical simulation is done using the MSC.ADAMS software. A prototype of the exoskeleton is manufactured and subjected to experimental tests. Finally, a comparison of motion laws performed by a healthy human subject and by the mannequin-exoskeleton assembly is made.

\section{Human gait experimental analysis}

Human gait analysis represents a large interest subject in the literature (Varela et al., 2015). Also experimental characterization of human falling down, represent a subject presented in recent studies (Meng et al., 2017). According to some studies (Winter et al., 1979; Tarnita et al., 2013), human gait represents a cyclic motion between heel strike on ground and next ground contact of same heel. This cycle consists of two important phases, stance phase and swing phase. Intermediate phases are also studied, a complete gait cycle phases being described by Perry et al. (2010).

For human gait experimental analysis, a Biometrics data acquisition system based on electrogoniometers is used (http: //www.biometricsltd.com/, Tarnita, 2016). Because are ro- 


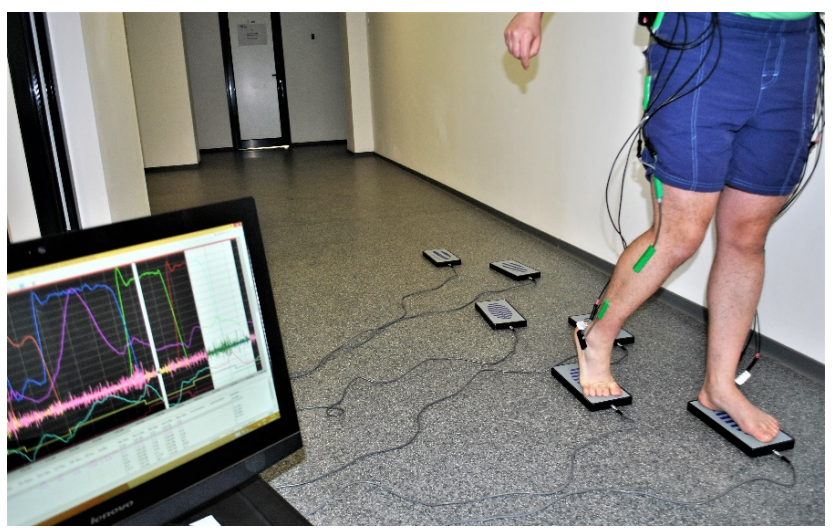

Figure 1. Goniometers and Datalog devices mounted on the human subject, during the test.

bust, lightweight and flexible, these sensors can be worn by subjects without alteration of the movement of the joint. Twin axis goniometers SG series are used to measure joint angles simultaneously in two planes of movement. For example, to measure knee movement, the end blocks of the goniometer are attached on the subject using double sided tape. The goniometer has two separate output connectors, one of them is used to measure knee flexion/extension angle and the other connector measures valgus/varus angle. For this research the measurement of flexion-extension angles in sagittal plane presents interest. In Fig. 1, the acquisition system composed by Biometrics software installed on the notebook, by electrogoniometers sensors and by the Datalog devices mounted on the human subject, is shown. The sensors are connected to Biometrics DataLog equipment through which the data are transferred to the computer via Bluetooth interface. Measurement frequency is specified to 500 data registration/second. The measurement accuracy of the goniometers is $\pm 2^{\circ}$ measured over a range of $\pm 90^{\circ}$ and the repeatability is $1^{\circ}$, measured over a range of $90^{\circ}$ (http://www.biometricsltd.com/gonio.htm).

The block schema of the acquisition system is shown in Fig. 2.

Experimental data acquired from a 35 years old healthy subject for normal walking during $30 \mathrm{~s}$ are reported as angle variation in time, for the ankle, knee and hip joints, in sagittal plane, for the right leg (Fig. 3) and for the left leg (Fig. 4). Experimental tests performed by the male subject consist in 25 consecutive gait cycles repeated for five times in the same walking conditions.

The anthropometric data of the human subject are: body weight $=700 \mathrm{~N}$, height $=1.67 \mathrm{~m}$, hip-knee length $=0.42 \mathrm{~m}$, knee-ankle length $=0.39 \mathrm{~m}$, ankle-little toe $=0.18 \mathrm{~m}$. The healthy subject was pain-free and had no evidence or known history of motor and skeletal disorders or record of surgery to the lower limbs. The study was approved by the Ethics Committee of Human Research, University of Craiova, Romania.
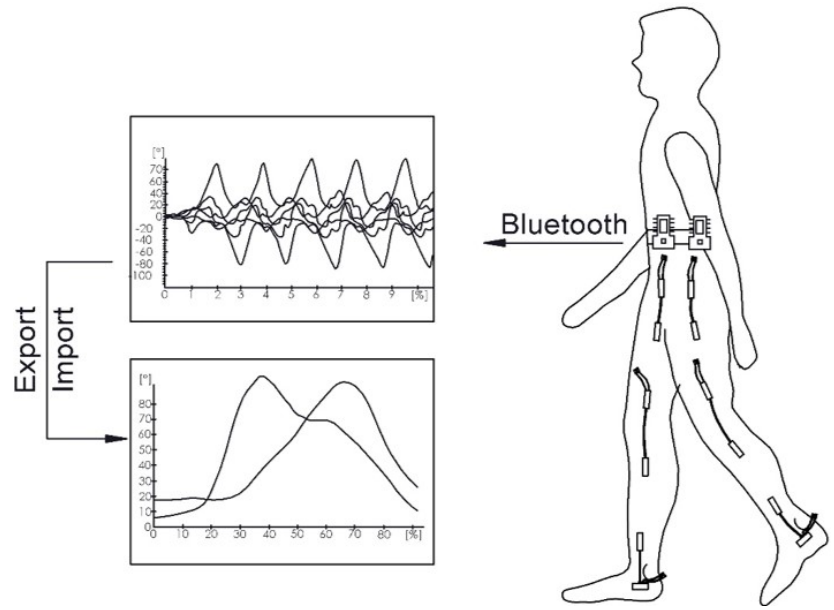

Figure 2. Block schema of the acquisition system.

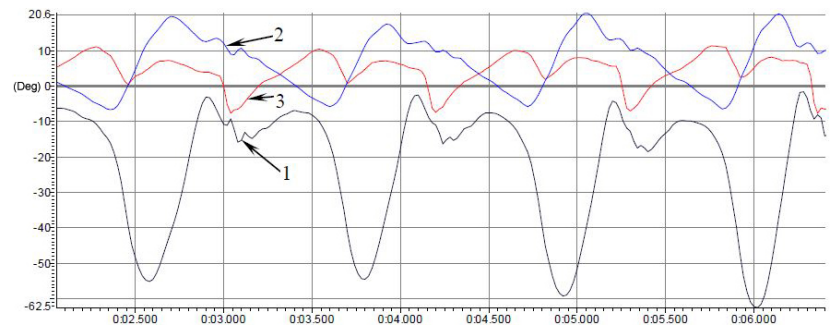

Figure 3. 1 - right knee flexion/extension; 2 - right hip flexion/extension; 3 - right ankle flexion/extension; Healthy human measured hip, knee and ankle joint angle, in sagittal plane, for right leg.

The knee joint angular maximum amplitude is by $65^{\circ}$ (Figs. 3-4) and for the hip joint the maximum amplitude is by $40^{\circ}$ (Figs. 3-4). These results are useful in order to make a comparison between achieved joints angles of human subject and joints angles achieved by the exoskeleton. According to Zoss et al. (2005), for human walking the hip flexion reaches a maximum value of $32.2^{\circ}$ and for knee $73.5^{\circ}$. Gait outputs of joint angles are presented by Lu et al. (2012). Results presented reveal that for healthy subject the hip flexion/extension varies between $\left[-15,25^{\circ}\right]$ and for the knee is comprised in the interval $\left[5,65^{\circ}\right]$. As a conclusion, human gait outputs presents variability from one subject to another. The results obtained in this research, for the human hip and knee joint flexion-extension angles, are comparable with those presented by other researchers in their studies.

The second set of experimental results is obtained, as data files, for the test of self-speed walking during $20 \mathrm{~s}$, from a disabled 50 years old male patient, suffering from osteoarthritis (OA) at left knee. The anthropometric data of the patient are: body weight $=730 \mathrm{~N}$, height $=1.65 \mathrm{~m}$, hipknee length $=0.41 \mathrm{~m}$, knee-ankle length $=0.38 \mathrm{~m}$, anklelittle toe $=0.16 \mathrm{~m}$. From Figs. 5-6, one can observe that the angular amplitude of the disabled patient knee joint differs 


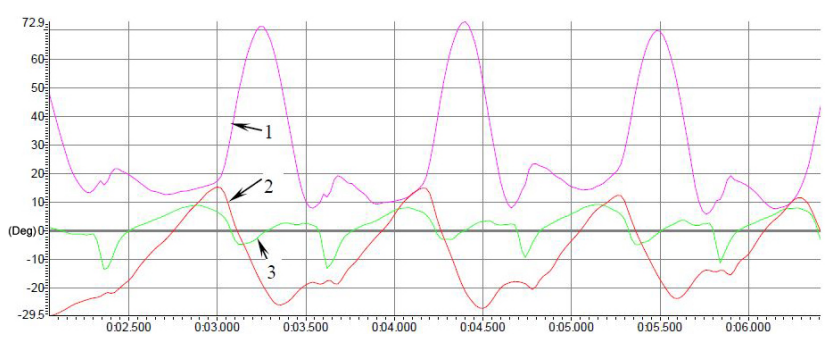

Figure 4. 1 - left knee flexion/extension; 2 - left hip flexion/extension; 3 - left ankle flexion/extension; Healthy human measured hip, knee and ankle joint angle, in sagittal plane, for left leg.

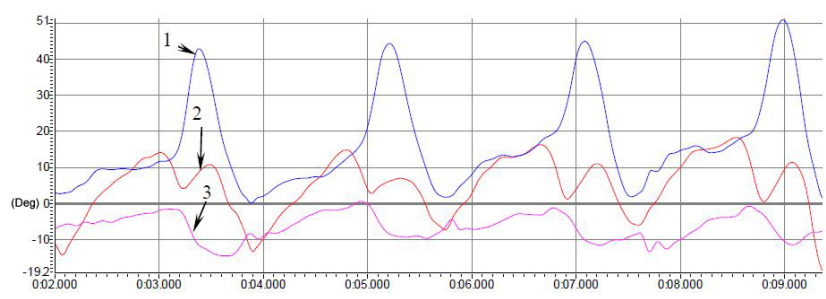

Figure 5. 1 - knee flexion/extension; 2 - hip flexion/extension; 3 ankle flexion/extension; The experimental angles variations for disabled human, in sagittal plane, for right leg.

from the healthy right joint to the osteoarthritic left knee joint. As we can see in Fig. 6, the maximum amplitude of flexion-extension cycles of OA knee is about $47^{\circ}$ and it is lower than the maximum amplitude of the unaffected right knee which is about $52^{\circ}$. The variation in time of the osteoarthritic knee angle (Fig. 6) is different from those of angle of the healthy right joint (Fig. 5), the patient dragging the disabled leg.

Human gait variability from one cycle to another for the same subject, from one subject to another and from one healthy subject to a diseased one, imposes the normalization of gait cycles. Normalization is done using SimiMotion software where the data files are transferred (Tarnita et al., 2017). For the accuracy of the final results, there were eliminated 2 cycles from the beginning and from end of data files. To compare results, it is necessary to determine the average cycle for each acquired data file.

\section{A new leg mechanism design}

Taking into account existing solutions of leg mechanisms used for human leg motion assistance and rehabilitation, presented in reviews and research studies, (Dollar et al., 2008; Rajesh et al., 2013; Bruzzone et al., 2012; Dumitru et al., 2015; Copilusi et al., 2015), for this research a single DOF, simple and light solution of a mechanism which assists the human gait is developed. There are developed similar one DOF leg solutions at LARM Laboratory Casino, Italy (Li et al., 2013; Wang et al., 2015b).

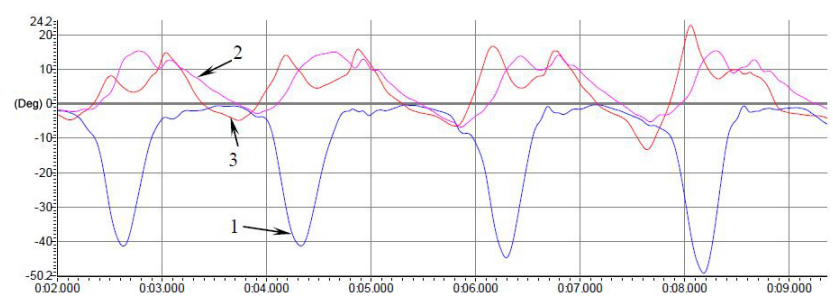

Figure 6. 1 - knee flexion/extension; 2 - hip flexion/extension; 3 ankle flexion/extension; The experimental angles variations for disabled human, in sagittal plane, for left leg (disabled leg).

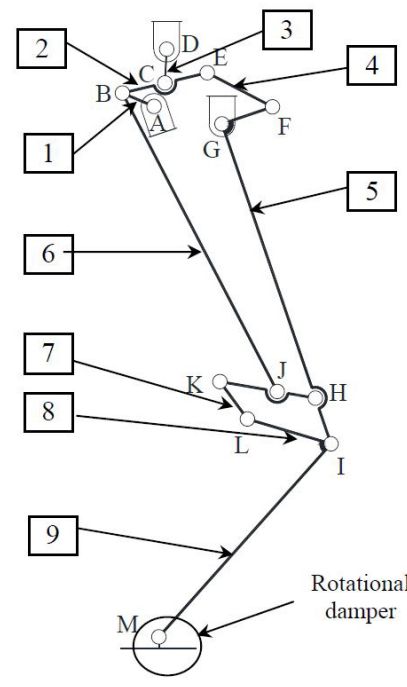

(a)

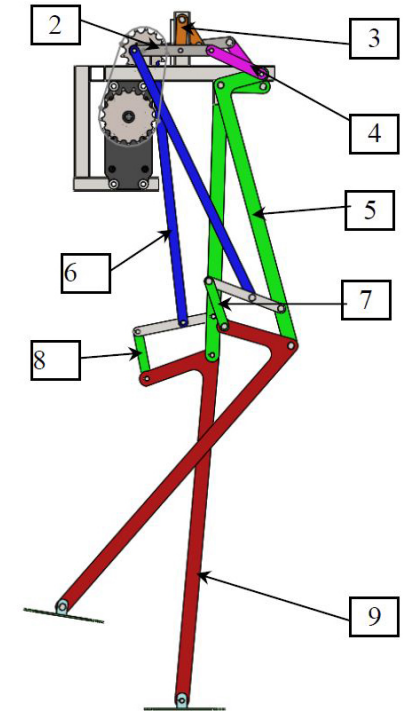

(b)
Figure 7. Leg exoskeleton: (a) a kinematic scheme; (b) virtual simulation model of leg exoskeleton.

The starting point in developing of a new leg mechanism is that the ankle joint trajectory must assure an ovoid path, to assure human leg motion during swing and also to assure proper angular variations of the joints. The proposed structural solution is optimized from kinematic perspective, using virtual modeling and simulation methods and principles (IIhem et al., 2013; Carbone et al., 2007) and path planning (Carbone et al., 2008; Tedeschi et al., 2015). The optimal design solution is materialized into an experimental prototype. The mechanical solution is completed with the command and control architecture. For actuation a DC gear motor is used. The motor speed is controlled with a hardware architecture based on pulse wave modulation method (PWM).

The structure of the leg exoskeleton and the virtual model designed in SolidWorks software package are presented in Fig. 7. This low cost solution uses only a rotary actuator mounted at the joint A of the link 1 which is fixed to the upper frame. The solution consists in a planar mechanism with 9 links and 13 revolute joints. The actuator rotates the link 1 of Cebyshev linkage 1-2-3. The femur link, 5, consists of 


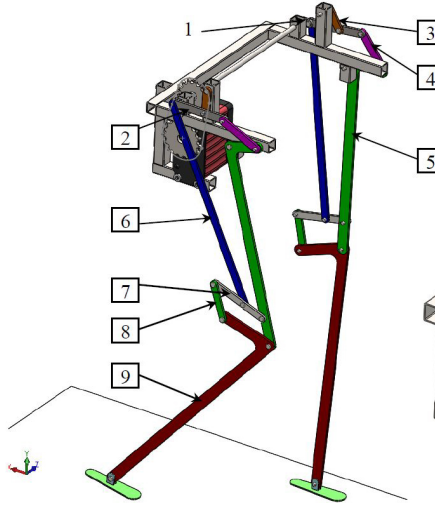

(a)

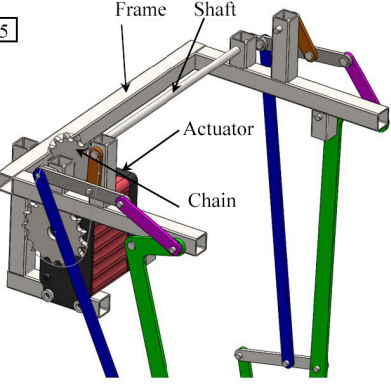

(b)
Figure 8. A virtual model of the exoskeleton in SolidWorks: (a) isometric view; (b) upper part detail view.

two orthogonal segments: FG and IG. Joint G, representing the hip, is connected to the upper frame, considered as a fixed element.

The link 4 transmits the motion, being connected by joint $\mathrm{E}$ to Chebyshev linkage, and by joint $\mathrm{F}$ to femur segment. Joint $\mathrm{B}$ is a multiple one, here being connected links 1 and 2 and link 6 with 1 . Through link 6 and the quadrilateral linkage ILKH the motion is transmitted from link 1 to the link 9 (materialized by exoskeleton tibia). Based on this innovative structure of the rehabilitation exoskeleton, a 3-D model is designed as it is presented in Fig. 8 .

In order to synthesize the lengths of the mechanism links we started from the patient dimensions (femur and tibia). The trajectory of ankle joint is tracked by performing 2D simulations in MSC.ADAMS (Wojtyra et al., 2003). Using ADAMS "Design of experiments" feature, a preliminary prototype optimization, using as constrains the femur and tibia lengths and the imposed hip and knee angular variation, is performed. For this case the mechanism operation is being carried on a supporting stand without contact with the ground. In this case, the design variables are the mechanism joints coordinates' variations. This innovative structure allows the leg exoskeleton to executes an ovoid path of the ankle joint M, (Fig. 9b), when it performs in stationary mode (the upper frame is fixed and the exoskeleton leg has not contact with ground), and it also allows proper variations of the angular amplitudes for the hip and knee joints noted $G$ and I, similar values with the experimental results presented in Figs. 3-4. Can be noticed in Fig. 7, that the ankle joint for this solution is not considered as an active one, the joint $\mathrm{M}$ being modeled as a rotational spring damper. As in case of other powered exoskeletons, the ankle joint is not actuated. The link (9) is connected with the exoskeleton foot (10), by a rotational joint $\mathrm{M}$. For the torsional spring are specified the rigidity and damping coefficients used to design the dynamic model of the exoskeleton.

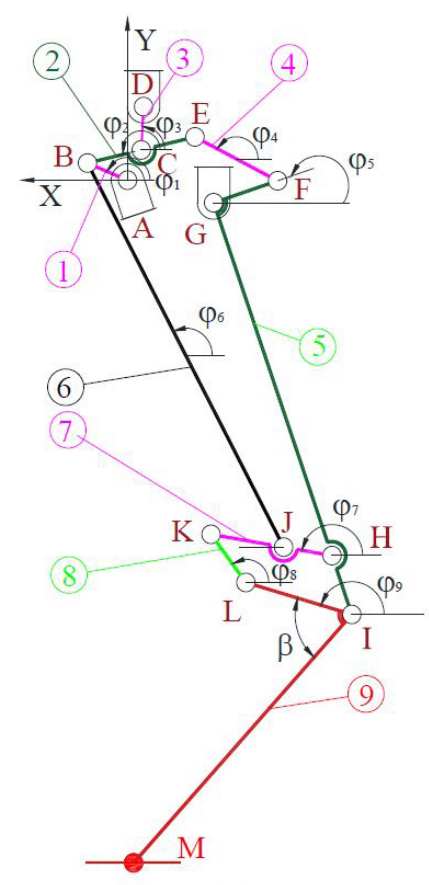

(a)

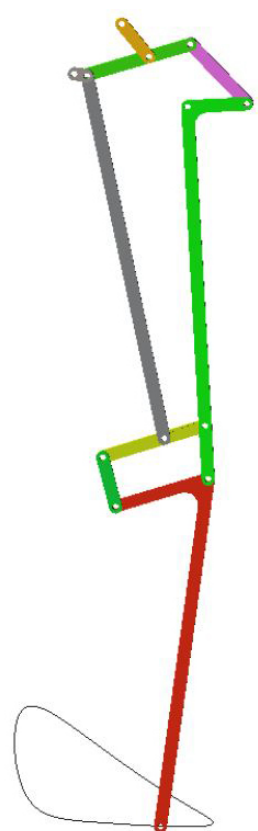

(b)
Figure 9. Mechanism for exoskeleton leg: (a) design parameters; (b) computed foot trajectory.

A virtual model of the exoskeleton assembly, consisting of two legs attached to an upper frame and of one chain transmission for both legs actuation, is designed in SolidWorks in order to perform a dynamic simulation. The links 1 of left and right legs are opposite positioned to $180^{\circ}$ taking into account the gait phases. In Fig. 8 is presented the exoskeleton assembly, comprising all of its components, according to the kinematic scheme presented in Fig. 7.

The proposed exoskeleton mechanism is characterized by some novel characteristics:

Comparing with other rehabilitation exoskeletons, the present proposed design has only one actuator. For that reason, it's a low-cost design solution and simple operation, because don't needs a complex command and control hardware.

The design solution can generate a suitable gait by its construction and gait planning is not necessary. By using a parallelogram in the knee joint, the foot moves parallel to the ground during propelling phase (as see in Fig. 9b, where is presented the computed ADAMS trajectory). Being a lowcost solution, the ankle joint is not actuated, but a torsion spring is mounted in order to be used for simulation.

It has an anthropomorphic structure, because the achieved joint motion of the exoskeleton is comparable with those of human subjects. A detailed comparison is presented in the paper.

The mechanism is characterized by simple operation. More, the exoskeleton is able to walk on inclined planes, because its structure assures a proper stride height. 


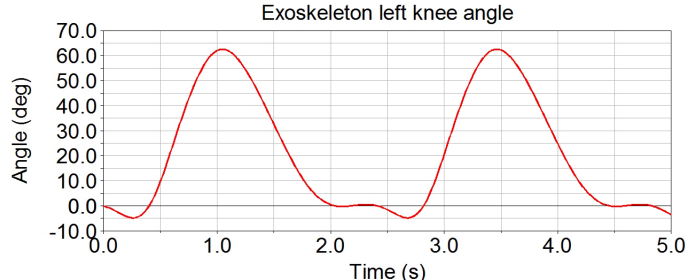

(a)

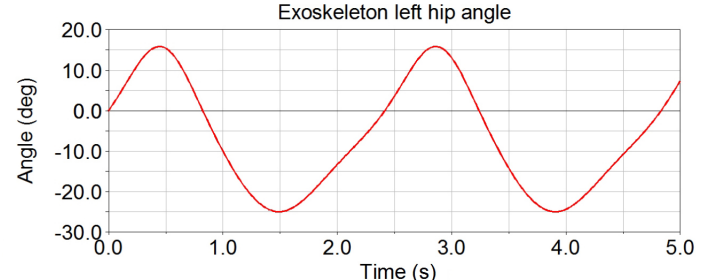

(b)

Figure 10. Computed joints angle variation: (a) knee joint; (b) hip joint.

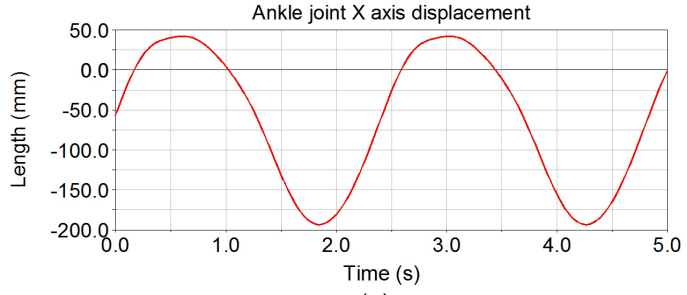

(a)

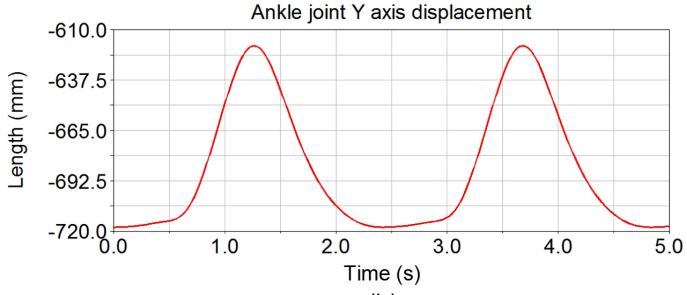

(b)

Figure 11. Computed plot of ankle joint M position: (a) upon $x$; (b) upon $y$ axis.

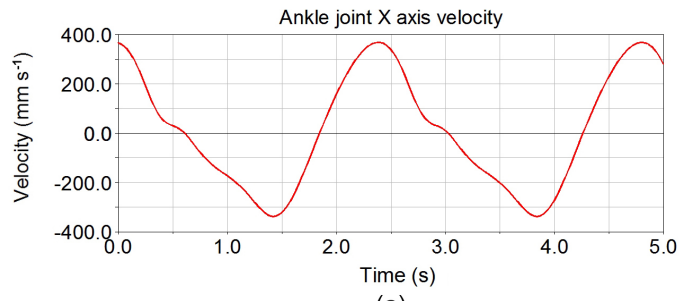

(a)

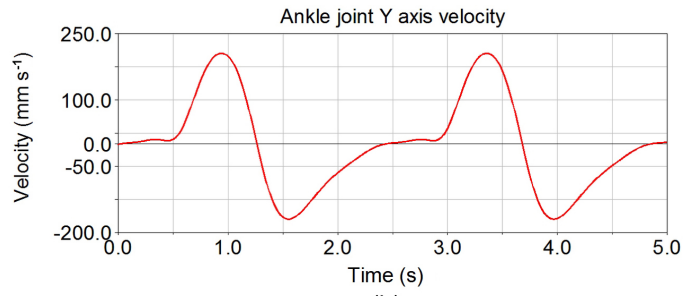

(b)

Figure 12. Computed plot of ankle joint M velocity: (a) upon $x$; (b) $y$ axis.

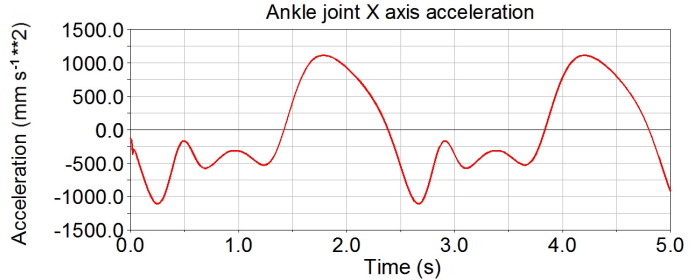

(a)

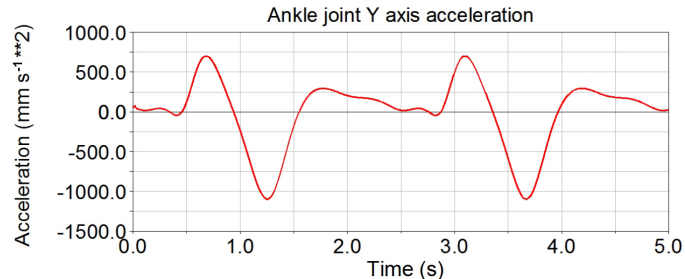

(b)

Figure 13. Computed plot of ankle joint $\mathrm{M}$ acceleration: (a) upon $x$ axis; (b) upon $y$ axis.

\section{Kinematics of exoskeleton leg mechanism}

In order to evaluate the prototype leg characteristics, a kinematic analysis considering the mechanism operation on a supporting stand is performed. The reference coordinate system XY has its origin placed in joint A. The design parameters of the leg mechanism are indicated in Fig. 9. The lengths of the mechanism elements and the coordinates of fixed joints A, D, G are shown in Table 1.

For a proper dimensional synthesis of the mechanism, the virtual simulation tools available in Solid Works software are used. The prototype of the exoskeleton is intended to be used by a young disabled person, with $1.58 \mathrm{~m}$ height. For this purpose, in order that the exoskeleton to be suitable to wear, are imposed the length of the segment GI to $350 \mathrm{~mm}$, that corresponds to the subject femur and the length of the segment IM to $315 \mathrm{~mm}$ corresponding to the tibia length. Another condition for the dimensional synthesis is to impose a minimum $90 \mathrm{~mm}$ horizontal distance between point $\mathrm{G}$ (hip joint) and joint A (where the engine shaft is located), allowing the exoskeleton to be suitable to wear by the patient (the patient must have sufficient space to fit with the pelvic basin). The 

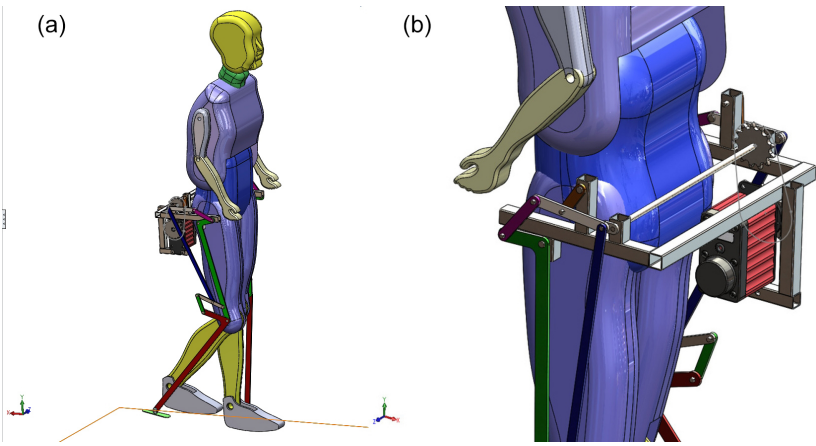

Figure 14. Exoskeleton virtual model worn by a human mannequin: (a) right leg view; (b) left leg back view.

link $A B$ is designed with a minimum dimension of $12 \mathrm{~mm}$ in the first phase for minimum power requirements and in final design with length of $13.5 \mathrm{~mm}$, in order to increase the knee and hip joints angular amplitude motion. Link BJ is designed with $355 \mathrm{~mm}$, because, in initial position, the segments GI and IM have to be a straight line, as the human leg in vertical position. Linkage IHKL is designed as a quadrilateral mechanism. Finally, the lengths of links 2, 3 and 4 are adjusted in SolidWorks parametric design, following to obtain an ovoid trajectory of the point $\mathrm{M}$ resulted by virtual simulation tracking.

The kinematics of the mechanism is described by the loop closure Eq. (1).

$$
\begin{aligned}
& l_{\mathrm{AB}}+l_{\mathrm{BC}}+l_{\mathrm{CD}}+l_{\mathrm{AD}}=0 ; l_{\mathrm{BE}}+l_{\mathrm{EF}}+l_{\mathrm{FG}}+l_{\mathrm{BG}}=0 \\
& l_{\mathrm{GH}}+l_{\mathrm{HJ}}+l_{\mathrm{JG}}=0 ; l_{\mathrm{KH}}+l_{\mathrm{HI}}+l_{\mathrm{IL}}+l_{\mathrm{KL}}=0
\end{aligned}
$$

Detailed equations of mechanism kinematics are presented in Appendix A.

The mathematical model characterizes the kinematics of the leg exoskeleton when it operates on a supporting stand. This kinematical model is solved in Maple software, and it is useful in order to validate the engineering feasibility of the proposed leg mechanism. Numerical results of the mathematical model are reported in plots in Figs. 10-13. In Fig. 10a, the computed variation of the knee angle is shown, and in Fig. 10b the computed variation of the hip angle is presented. By comparing the obtained motion laws with those obtained in experimental gait analysis, for the healthy subject, presented in Figs. 3-4, one can remark that the variation in time is almost similar. The numerical angular amplitude for the knee joint varies in the interval $\left[-4,64^{\circ}\right]$ with a maximum value equal to $68^{\circ}$, comparable to $65^{\circ}$, value obtained in the experimental gait analysis, presented in Figs. 3, 4. In the case of hip joint, the numerical angular amplitude varies in the interval $\left[-25,16^{\circ}\right]$ with a maximum amplitude equal to $41^{\circ}$. Also, the hip angular amplitude is close by $40^{\circ}$, similar with the one obtained for the human hip joint.

The leg exoskeleton realizes a displacement of $220 \mathrm{~mm}$, from -180 to $40 \mathrm{~mm}$, along horizontal $x$ axis, corresponding to walking direction in sagittal plane (Fig. 11a). The vertical displacement, corresponding to the step height, as shown in Fig. $11 \mathrm{~b}$ is about $110 \mathrm{~mm}$, from -720 to $-610 \mathrm{~mm}$. The negative values are explained because the reference coordinate system has the origin placed in A joint, as shown in Fig. 9a. The constant intervals of the variation shown in Fig. 11b corresponds to the propelling phase of the leg (push off), when the joint $\mathrm{M}$ achieves a linear trajectory. The diagrams of the point $\mathrm{M}$ displacements, representing the ankle joint motion, have the same allures to those experimentally obtained for human gait analysis by others researchers (Copilusi et al., 2015; Tarnita et al., 2013).

The walking velocities on $x$ and $y$ axes for the $\mathrm{M}$ joint range in the interval $[-320 ; 380] \mathrm{mm} \mathrm{s}^{-1}$, respectively, in the interval $[-180 ; 230] \mathrm{mm} \mathrm{s}^{-1}$, as shown in Fig. 12, while, the computed acceleration on $x$ and $y$ axes of the $\mathrm{M}$ joint, presented in Fig. 13, varies in the interval $[-1115 ; 1105] \mathrm{mm} \mathrm{s}^{-1}$, respectively, in the interval $[-1115$; 700] $\mathrm{mm} \mathrm{s}^{-2}$.

\section{Modeling and simulation}

A virtual model of the exoskeleton, consisting of two legs attached to an upper frame, and a chain transmission for actuation of the legs is designed in SolidWorks for dynamic simulation. The links 1 of left and right legs are opposite positioned to $180^{\circ}$. According to the kinematic scheme presented in Fig. 7, the exoskeleton design, comprising all of its components, is performed and presented in Fig. 14. The mannequin is modeled with all anatomical parts (trunk, arm and legs), and movable leg links (femur, tibia and foot) based on anthropomorphic data of the patient. The anatomical parts of mannequin are defined as geometry and inertial properties (by adding material density). The simulation system is defined by 25 revolute joints for the exoskeleton system, 6 revolute joints for the mannequin, and suitable fixed joints used in order to attach the exoskeleton to the mannequin. The chain transmission which has the role of rotating the shaft and the legs motor links 1 , is also designed. All the revolute joints of the system have been defined considering the Coulomb friction model, respectively: the static friction coefficient is considered equal to 0.1 and the dynamic coefficient is equal to 0.05 . In our design, in order to model a proper contact, the foot contact models were defined using two spheres: one for heel and one for plantar area, as we can see in Fig. 15b).

In the literature are available several studies on the importance of the foot-ground contact definition. Valiant (1990) published studies on the dynamic characteristics of the plantar surface of the foot. Then, this work has been continued and developed by Meglan et al. (1992), who studied the loaddeformation parameters of the foot-ground and developed a general equation to compute vertical ground reaction force, using optimization techniques in order to fit Valiant's data results to a simple polynomial equation. The obtained equation 


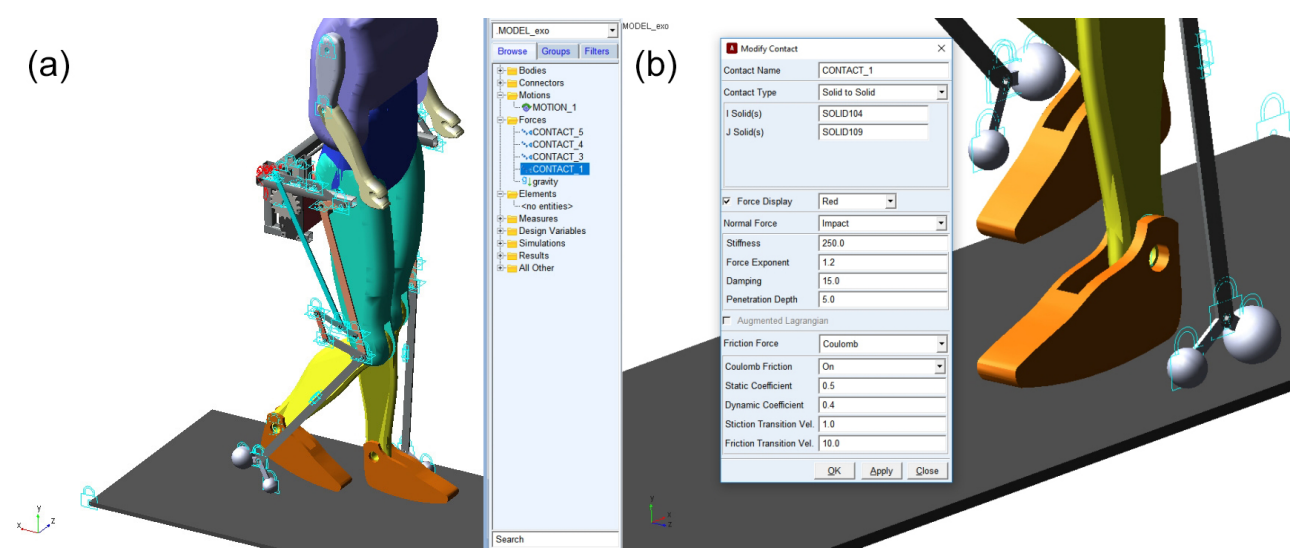

Figure 15. Dynamic model of leg exoskeleton for simulation: (a) ADAMS model; (b) foot ground contact in ADAMS.

Table 1. Design parameters of leg mechanism.

\begin{tabular}{l|l|l}
\hline$l_{1}=l_{\mathrm{AB}}=13.5 \mathrm{~mm}$ & $l_{4}=l_{\mathrm{EF}}=72 \mathrm{~mm}$ & $l_{\mathrm{GH}}=300 \mathrm{~mm}$ \\
$l_{\mathrm{BC}}=40 \mathrm{~mm}$ & $l_{6}=l_{\mathrm{BJ}}=355 \mathrm{~mm}$ & $l_{\mathrm{GI}}=350 \mathrm{~mm}$ \\
$l_{3}=l_{\mathrm{CD}}=40 \mathrm{~mm}$ & $l_{8}=l_{\mathrm{KL}}=48 \mathrm{~mm}$ & $l_{\mathrm{HJ}}=40 \mathrm{~mm}$ \\
$l_{\mathrm{CE}}=60 \mathrm{~mm}$ & $l_{\mathrm{FG}}=55 \mathrm{~mm}$ & $l_{\mathrm{JK}}=60 \mathrm{~mm}$ \\
$l_{\mathrm{IL}}=90 \mathrm{~mm}$ & $x_{\mathrm{A}}=0, y_{\mathrm{A}}=0 \mathrm{~mm}$ & Step length $=220 \mathrm{~mm}$ \\
$l_{\mathrm{IM}}=315 \mathrm{~mm}$ & $x_{\mathrm{D}}=-33.64 \mathrm{~mm}, y_{\mathrm{D}}=45.5 \mathrm{~mm}$ & Step height $=110 \mathrm{~mm}$ \\
$l_{\mathrm{IH}}=50 \mathrm{~mm}$ & $x_{\mathrm{G}}=-90.7 \mathrm{~mm}, y_{\mathrm{G}}=-32 \mathrm{~mm}$ & $\beta=65^{\circ}$ \\
\hline
\end{tabular}

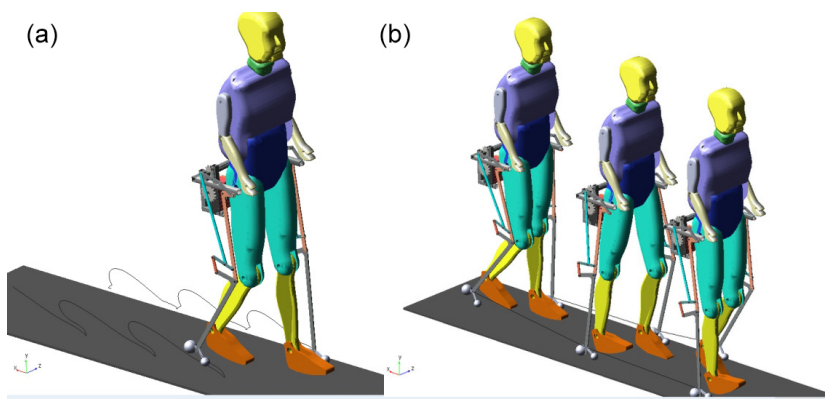

Figure 16. Snapshots of simulation outputs of the exoskeleton and mannequin walking.

was (Patton et al., 1993):

$F(x, v)=\left(4.658 \times 10^{10}\right) x^{4}+\left(9.719 \times 10^{10}\right) x^{4} \cdot v$

where: $F$ is the resulting contact force, $x$ is the penetration depth and $v$ is the velocity of penetration. The ADAMS contact modeling tools do not allow for such a polynomial function to be introduced in the model, but offer instead the following cubic function (with IMPACT method used to define the contact), (Patton et al., 1993):

$F(x, v)=k x+\left\{c(x / d)^{2} \times[3-2 x / d]\right\} v$

where: $k$ is a linear spring constant, $c$ is the damping coefficient and $d$ is the depth of penetration. The estimates of the ADAMS impact coefficients, upon Meglan relations are: $k=250 \mathrm{~N} \mathrm{~mm}^{-2} ; c=15 \mathrm{~N} \mathrm{~mm}^{-1} \mathrm{~s}^{-1}$ and $d=5 \mathrm{~mm}$. The exoskeleton contact parameters and also designed foot model are shown in Fig. 15.

The torsion spring damper, placed at ankle joint uses the coefficients: $\left(K_{\text {rot }}\right)=1 \mathrm{~N} \mathrm{rad}^{-1}-$ representing the stiffness coefficient and $\left(C_{\text {rot }}\right)=1 \mathrm{~N} \mathrm{~mm} \mathrm{rad}{ }^{-1}-$ the damping coefficient (Kesckemethy et al., 2011).

The simulation settings for the mannequin - exoskeleton assembly were considered as real. The model of the mannequin was positioned in the bipedal position when the left foot touches the ground and the right leg is in the balancing phase. This is the same starting position of the man subjected to experimental walking analysis. Simulation of the exoskeleton in MSC.ADAMS is proposed for the case of assistance to a patient with a leg disability. A sequence of exoskeleton with mannequin walking positions is shown in Fig. 16.

For the simulation, input angular velocity is set up to $4 \mathrm{rad} \mathrm{s}^{-1}$. When the motor rotates $180^{\circ}$, the bipedal mechanism moves one step forward.

Numerical results of the mannequin - exoskeleton assembly gait simulation are presented in the plots from Figs. 1722 , related to the snapshots of virtual simulation, shown in Fig. 16. When the exoskeleton is worn by a human mannequin and performs gait, the obtained numerical results for the hip and knee joints angular variation are shown in Fig. 17. Presented plots show that the computed angular amplitude of 


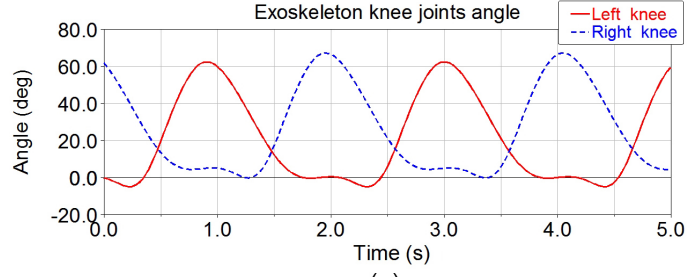

(a)

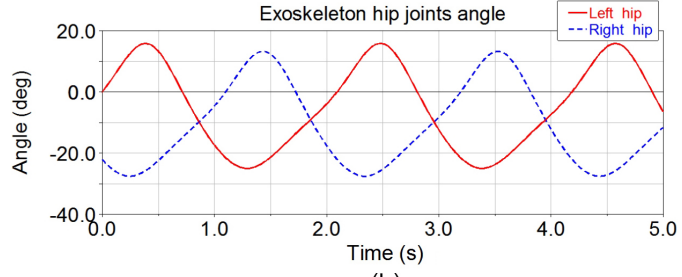

(b)

Figure 17. Computed exoskeleton joints angle (dotted line-right leg, continuous line-left leg): (a) knee joints; (b) hip joints.

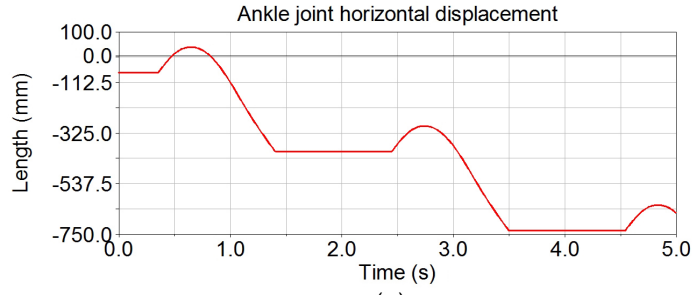

(a)

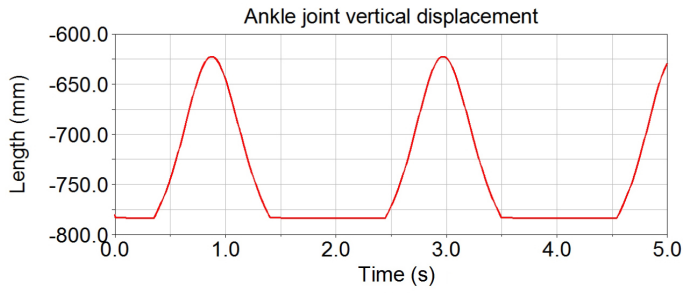

(b)

Figure 18. Computed displacement of the left leg exoskeleton ankle joint: (a) on $x$ axis; (b) on $y$ axis.

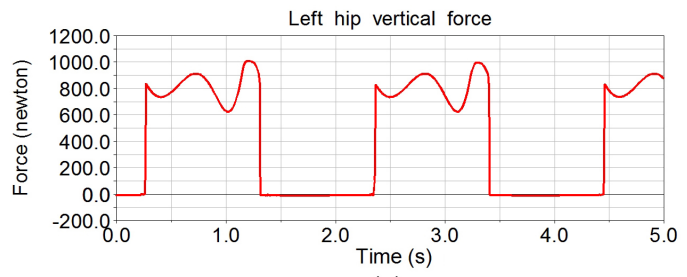

(a)

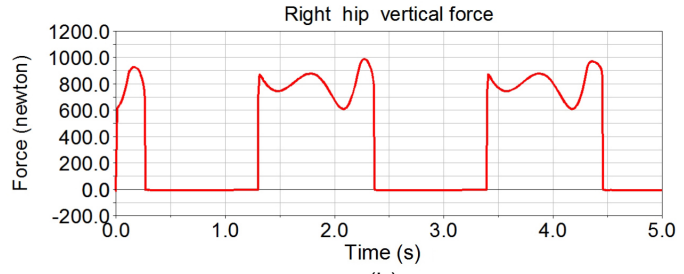

(b)

Figure 19. Computed vertical reaction force on the exoskeleton hip joint: (a) left joint; (b) right joint.

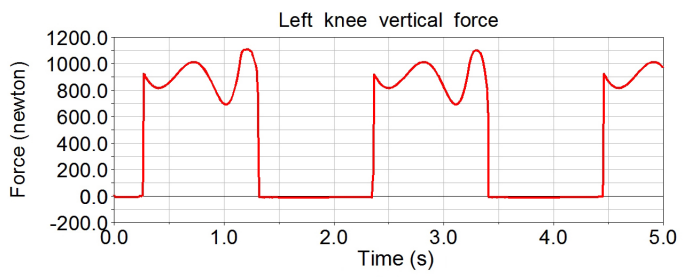

(a)

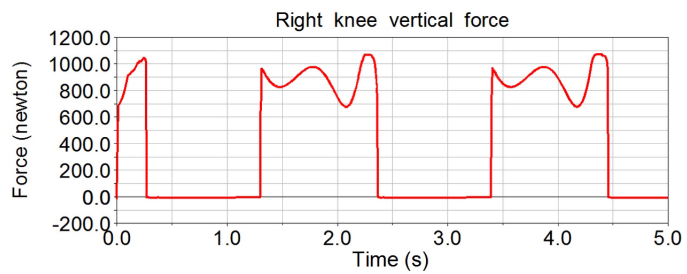

(b)

Figure 20. Computed vertical reaction force on the exoskeleton knee joint: (a) left knee; (b) right knee.

the right hip joint vary in the range $\left[-25 ; 16^{\circ}\right]$, with a maximum amplitude equal to $41^{\circ}$, while for the right knee joint the computed angular amplitude vary in the range $\left[0 ; 65^{\circ}\right]$ and the maximum amplitude is $65^{\circ}$. The values of computed amplitudes are similar with the ones obtained by experimental analysis, presented in Figs. 3 and 4.

Figure 18 shows computed displacements of the left leg exoskeleton ankle joint, upon horizontal axis Fig. 18a and vertical axis Fig. 18b. In the first phase the leg is on the ground during $1.5 \mathrm{~s}$, and then the leg is performing the swing phase, with a step length by $440 \mathrm{~mm}$. The step length performed when the exoskeleton is performing gait is bigger than in situation when the mechanism operates at stationary with $220 \mathrm{~mm}$ (is double). This fact is confirmed also by the experimental analysis of the exoskeleton gait.

Figures 19 and 20 show the reaction forces calculated at the hip and knee joints of the exoskeleton. The vertical reaction force reaches a maximum of $1000 \mathrm{~N}$ for the hip joint and $1100 \mathrm{~N}$ for the knee joint. The weight of the mannequin - exoskeleton assembly is $950 \mathrm{~N}$, so that the horizontal force component of the exoskeleton knee is approximately 1.15 times the weight of the model. The vertical reaction forces computed reaches the maximum amplitude when the exoskeleton leg it detaches from the ground. Figure 21 shows 


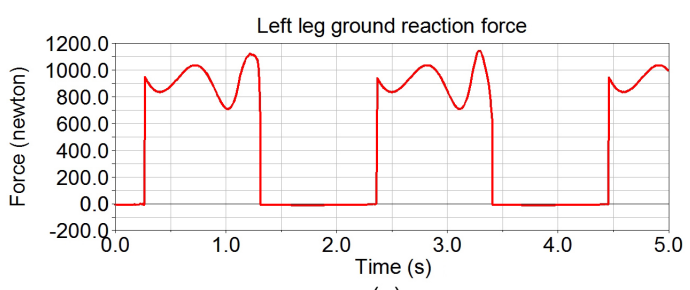

(a)

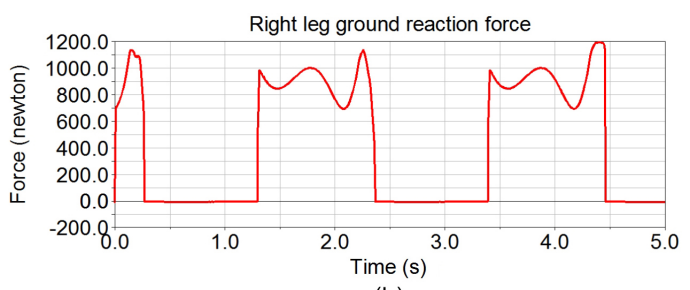

(b)

Figure 21. Computed exoskeleton ground reaction forces (GRF): (a) for left leg; (b) for right leg.

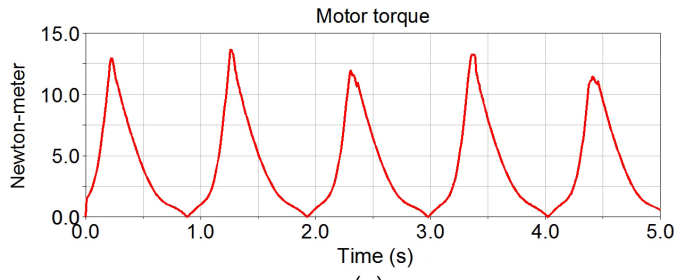

(a)

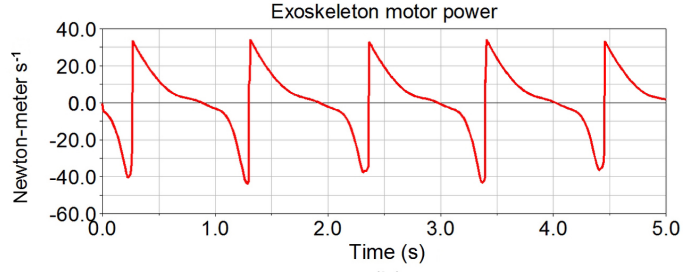

(b)

Figure 22. Computed actuator parameters: (a) $z$ axis motor torque; (b) motor power magnitude.
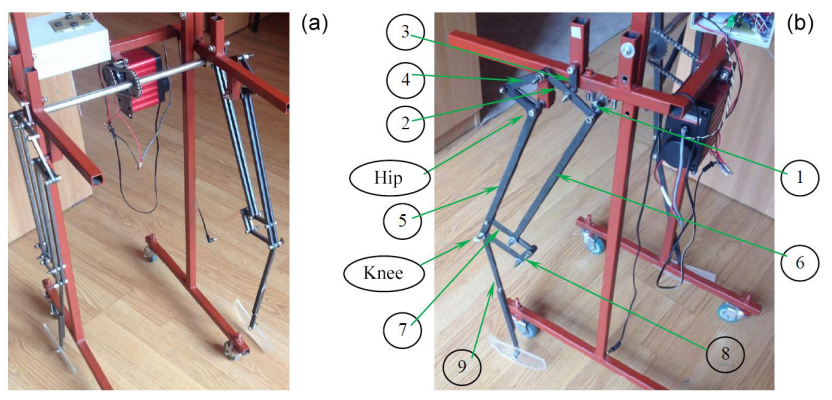

Figure 23. A view of exoskeleton manufactured prototype: (a) isometric view; (b) lateral view.

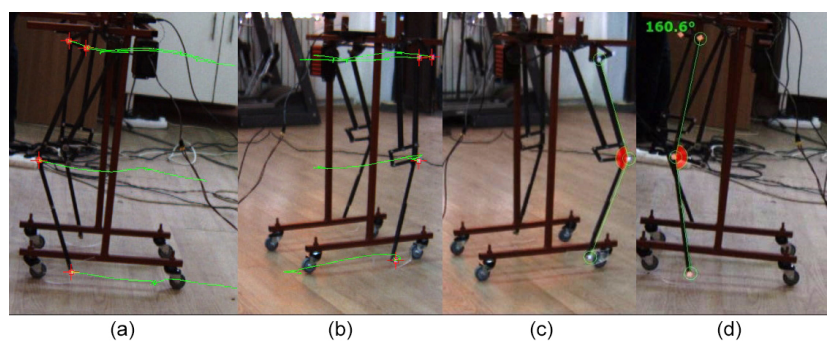

Figure 24. Marker tracking for exoskeleton kinematics: (a, b) computation of trajectories; $(\mathbf{c}, \mathbf{d})$ computation of knee joints angular variation.

the exoskeleton ground reaction forces for both legs. The maximum value is $1130 \mathrm{~N}$, also a value correlated with the weight of the assisted virtual mannequin (patient). Figure 22 shows computed torque of driving motor on rotation axis and computed power of the actuator. As is observed the time for a step is about $1.5 \mathrm{~s}$, and the time period of one walking op- eration cycle (two steps) is $3 \mathrm{~s}$. The peaks values are $14 \mathrm{Nm}$, corresponding to the ground contact phase of the legs. For the swing phase of the exoskeleton the values for the forces and computed torque are smaller. Computed diagrams for the legs show repetitive variations. Simulation results allow concluding that the leg exoskeleton design is suitable for human gait rehabilitation.

\section{Experimental characterization of the exoskeleton}

The proposed exoskeleton design is subjected to experimental tests, to validate the dynamic simulation. For that purpose, based on the optimal design, the experimental prototype of the exoskeleton is manufactured (Fig. 23). For the exoskeleton actuation, a DC electric motor is used. The electric motor design uses a gearbox in order to deliver a high torque at a low rotational output. In addition, the exoskeleton design uses a chain transmission, which can be customizable to adopt different supplementary transmission ratios. The maximum torque delivered by the motor is $15 \mathrm{Nm}$ at $38 \mathrm{rpm}$ rated speed. The motor is powered by a 12 volts accumulator. To adjust the electric motor speed, a pulse wave modulation (PWM) controller is implemented based on an Arduino board. In first stage of experimental tests, the exoskeleton walks without a human subject. During the walking, the exoskeleton stability is guaranteed by two links, which run on the ground with two self-directional wheels. In this way there are assured two additional ground contact points in order to have dynamic stability conditions. The walking of the exoskeleton is performed on laboratory floor, and the motion is analyzed based on video cameras motion analysis equipment CONTEMPLAS. Reflective markers on different inter- 

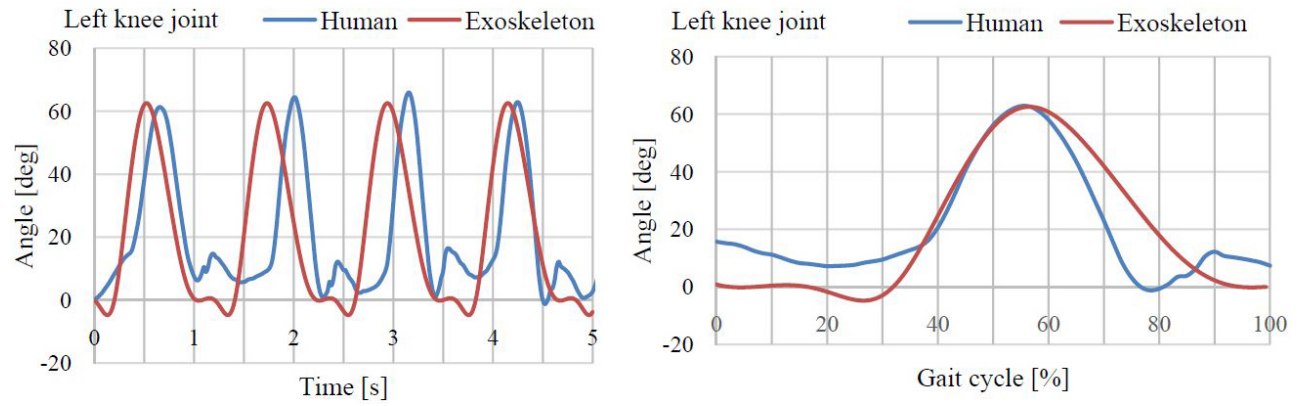

Figure 25. Compared variation of knee joint angle for human subject and for exoskeleton model.
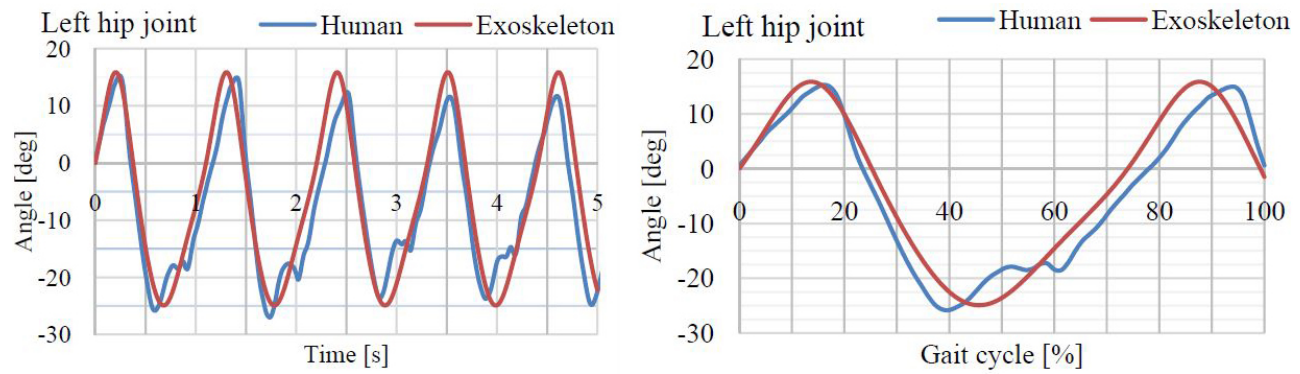

Figure 26. Compared variation of hip joint angle for human subject and for exoskeleton model.

est points, like hip, knee and ankle joint are attached and their captured motion trajectories are shown in Fig. 24a and b.

Joints angle variation is computed based on marker tracking (Fig. 24c, d). Numerical results, obtained for the exoskeleton joint variations are compared with those achieved by the human subject. For the human subject hip and knee joint angular variation a medium cycle is calculated.

A comparison of the achieved left knee joint angular variation for the exoskeleton and human healthy subject is presented in Fig. 25. Also because of the human gait variability for a relevant comparison are presented on the same diagram the variations for the exoskeleton knee joint and the human subject medium cycle, for a gait cycle. It can be observed that the maximum angular amplitude for the human and exoskeleton knee joint is $65^{\circ}$. Because the human gait presents variability from one step to another and from one subject to another, one can observe in Fig. 25 that the period corresponding to each step made by the human subject differs from one to other. The steps performed by the exoskeleton do not present variability. For the comparison the medium cycle of the human subject is used. For more accurate results, considering the natural biological variability from one's individual step to another, 19 consecutive cycles of flexion-extension angle were selected (after removing the first three cycles and the last three cycles), and, then, were normalized by interpolation in SimiMotion software and reported on the abscissa at a scaled interval from 0 to $100 \%$. For each trial the mean cycle was obtained, and, finally, the mean cycle of the experimental test was obtained based on the five mean cycles corresponding to the five trials.

By comparing the five maximum values of the mean cycles of knee flexion-extension with the maximum value of the final mean cycle corresponding to the test, we can conclude that, these are very close with non-significant differences. There were not big differences in the shape of the flexion angle. The minor differences obtained by this comparison show a good repeatability of the imposed test for all the five trials. The maximum values of the knee angle determined during the performed trials were compared and tested with a Student $t$-test, considering $\alpha=0.05$. The $\mathrm{P}$-values corresponding to these tests are calculated using ANOVA. The conclusion is that the maximum flexion angles were not significantly different $\left(t_{\text {calc }}=2.09<t_{\mathrm{cr}}=2.31\right.$ and $\left.p=0.0785>0.05\right)$.

The angular variations diagrams in case of human hip joint and exoskeleton hip joint are presented in Fig. 26. For the hip joint, the angular variation achieved by the human and exoskeleton is between $\left[-25^{\circ} ; 15\right]$. One can observe that in both cases (human and exoskeleton), the shapes of the compared graphics is similar. 


\section{Conclusions}

A new exoskeleton mechanism, including the design model, the simulation results and the experimental prototype, is presented. The proposed exoskeleton is designed based on a low cost and easy-to-use design. The functionality of the exoskeleton is studied for the case when it is worn by a virtual human mannequin. For that purpose a dynamic simulation model in ADAMS is developed. The results obtained by numerical simulation are discussed and compared with experimental results obtained on healthy human subjects. Based on the CAD design an experimental prototype of the exoskeleton is manufactured in order to perform experimental studies and to validate the results obtained by numerical simulation. The exoskeleton real model motion analysis is performed using Contemplas ultra speed video cameras equipment and analysis software. The motion of the experimental prototype is compared with results of virtual simulation motion and with results of the human gait experimental analysis. Obtained results are used to characterize the operation of the leg proposed exoskeleton. Finally is presented a comparison of exoskeleton motion compared results. The exoskeleton hip and knee joints angular motion it is compared with human healthy subject motion and the obtained variation graphics are similar. In conclusion, the mechanism operation is suitable for human motion assistance and rehabilitation purposes.

Data availability. All the data used in this manuscript can be obtained by requesting from the corresponding author. 


\section{Appendix A: Mechanisms kinematics equations}

Equations (1) are projected on the coordinate system axis, and the Eqs. (A1)-(A4) are obtained, where the unknowns are the angles $\varphi_{i}, i=\overline{2,9}$.

$\left\{\begin{array}{l}x_{\mathrm{B}}+l_{\mathrm{BC}} \cdot \cos \varphi_{2}=x_{\mathrm{D}}+l_{3} \cdot \cos \varphi_{3} \\ y_{\mathrm{B}}+l_{\mathrm{BC}} \cdot \sin \varphi_{2}=y_{\mathrm{D}}+l_{3} \cdot \sin \varphi_{3}\end{array}\right.$
$\left\{\begin{array}{l}x_{\mathrm{E}}+l_{4} \cdot \cos \varphi_{4}=x_{\mathrm{G}}+l_{\mathrm{GF}} \cdot \cos \varphi_{5} \\ y_{\mathrm{E}}+l_{4} \cdot \sin \varphi_{4}=y_{\mathrm{G}}+l_{\mathrm{GF}} \cdot \sin \varphi_{5}\end{array}\right.$
$\left\{\begin{array}{l}x_{\mathrm{B}}+l_{\mathrm{BJ}} \cdot \cos \varphi_{6}=x_{\mathrm{H}}+l_{\mathrm{JH}} \cdot \cos \varphi_{7} \\ y_{\mathrm{B}}+l_{\mathrm{BJ}} \cdot \sin \varphi_{6}=y_{\mathrm{H}}+l_{\mathrm{JH}} \cdot \sin \varphi_{7}\end{array}\right.$
$\left\{\begin{array}{l}x_{\mathrm{K}}+l_{8} \cdot \cos \varphi_{8}=x_{\mathrm{I}}+l_{\mathrm{L}} \cdot \cos \varphi_{9} \\ y_{\mathrm{K}}+l_{8} \cdot \sin \varphi_{8}=y_{\mathrm{I}}+l_{\mathrm{IL}} \cdot \sin \varphi_{9}\end{array}\right.$

Solutions of Eqs. (A1)-(A4) are found by solving nonlinear Eq. (A5), with variable coefficients. These loop closure equations are solved using a package program developed on Maple environment.

$$
\begin{aligned}
& A_{i} \sin \varphi_{i}+B_{i} \cos \varphi_{i}+C_{i}=0 ; i=\overline{2,9} . \\
& \varphi_{i}=2 \operatorname{arctg}\left(\frac{A_{i} \pm \sqrt{A_{i}^{2}+B_{i}^{2}-C_{i}^{2}}}{B_{i}-C_{i}}\right)
\end{aligned}
$$

where the variable coefficients $A_{i}, i=\overline{2,9}$, are computed with Eq. (A6):

$$
\begin{aligned}
& A_{2}=2 b_{1} l_{\mathrm{BC}} ; B_{2}=2 a_{1} l_{\mathrm{BC}} ; C_{2}=l_{3}^{2}-a_{1}^{2}-b_{1}^{2}-l_{\mathrm{BC}}^{2} ; \\
& A_{3}=-2 b_{1} l_{3} ; B_{3}=-2 a_{1} l_{3} ; C_{3}=l_{\mathrm{BC}}^{2}-a_{1}^{2}-b_{1}^{2}-l_{3}^{2} \\
& A_{4}=2 b_{2} l_{4} ; B_{4}=2 a_{2} l_{4} ; C_{4}=l_{\mathrm{GF}}^{2}-l_{4}^{2}-a_{2}^{2}-b_{2}^{2} ; \\
& A_{5}=-2 b_{2} l_{\mathrm{GF}} ; B_{5}=-2 a_{2} l_{\mathrm{GF}} ; C_{5}=l_{4}^{2}-l_{\mathrm{GF}}^{2}-a_{2}^{2}-b_{2}^{2} \\
& A_{6}=2 c_{2} l_{\mathrm{BJ}} ; B_{6}=2 c_{1} l_{\mathrm{BJ}} ; C_{6}=l_{\mathrm{JH}}^{2}-c_{1}^{2}-c_{2}^{2}-l_{\mathrm{BJ}}^{2} ; \\
& A_{7}=-2 c_{2} l_{\mathrm{JH}} ; B_{7}=-2 c_{1} l_{\mathrm{JH}} ; C_{7}=l_{\mathrm{BJ}}^{2}-c_{1}^{2}-c_{2}^{2}-l_{\mathrm{JH}}^{2} \\
& A_{8}=2 d_{2} l_{8} ; B_{8}=2 d_{1} l_{8} ; C_{8}=l_{\mathrm{I}}^{2}-d_{1}^{2}-d_{2}^{2}-l_{8}^{2} ; \\
& A_{9}=-2 d_{2} l_{\mathrm{IL}} ; B_{9}=-2 d_{1} l_{\mathrm{IL}} ; C_{9}=l_{\mathrm{IL}}^{2}-d_{1}^{2}-d_{2}^{2}-l_{8}^{2} \\
& \text { where } \\
& a_{1}=x_{\mathrm{D}}-x_{\mathrm{B}}, b_{1}=y_{\mathrm{D}}-y_{\mathrm{B}} ; a_{2}=x_{\mathrm{G}}-x_{\mathrm{E}}, b_{2}=y_{\mathrm{G}}-y_{\mathrm{E}} \\
& c_{1}=x_{\mathrm{H}}-x_{\mathrm{B}}, c_{2}=y_{\mathrm{H}}-y_{\mathrm{B}} ; d_{1}=x_{\mathrm{I}}-x_{\mathrm{K}}, d_{2}=y_{\mathrm{I}}-y_{\mathrm{K}}
\end{aligned}
$$

The position of point $\mathrm{M}$ can be evaluated as an input crank angle function, Eq. (A7):

$$
\begin{aligned}
x_{\mathrm{M}} & =l_{1} \cdot \cos \varphi_{1}+l_{\mathrm{BE}} \cdot \cos \varphi_{2}+l_{4} \cdot \cos \varphi_{4}+l_{\mathrm{FG}} \cdot \cos \varphi_{5} \\
& +l_{\mathrm{GI}} \cdot \cos \left(\varphi_{5}+\pi / 2\right)+l_{I M} \cdot \cos \left(2 \pi-\beta+\varphi_{9}\right) \\
y_{\mathrm{M}} & =l_{1} \cdot \sin \varphi_{1}+l_{\mathrm{BE}} \cdot \sin \varphi_{2}+l_{4} \cdot \sin \varphi_{4}+l_{\mathrm{FG}} \cdot \sin \varphi_{5} \\
& +l_{\mathrm{GI}} \cdot \sin \left(\varphi_{5}+\pi / 2\right)+l_{\mathrm{IM}} \cdot \sin \left(2 \pi-\beta+\varphi_{9}\right)
\end{aligned}
$$

The components of the point $\mathrm{M}$ velocity on both axes are given by the Eq. (A8):

$$
\begin{aligned}
v_{x \mathrm{M}}= & -l_{1} \cdot \sin \varphi_{1} \cdot \omega_{1}-l_{\mathrm{BE}} \cdot \sin \varphi_{2} \cdot \omega_{2}-l_{4} \cdot \sin \varphi_{4} \cdot \omega_{4} \\
& -l_{\mathrm{FG}} \cdot \sin \varphi_{5} \cdot \omega_{5}-l_{\mathrm{GI}} \cdot \sin \left(\varphi_{5}+\pi / 2\right) \cdot \omega_{5} \\
& -l_{\mathrm{IM}} \cdot \sin \left(2 \pi-\beta+\varphi_{9}\right) \cdot \omega_{9} \\
v_{y \mathrm{M}}= & l_{1} \cdot \cos \varphi_{1} \cdot \omega_{1}+l_{\mathrm{BE}} \cdot \cos \varphi_{2} \cdot \omega_{2}+l_{4} \cdot \cos \varphi_{4} \cdot \omega_{4} \\
& +l_{\mathrm{FG}} \cdot \cos \varphi_{5} \cdot \omega_{5}+l_{\mathrm{GI}} \cdot \cos \left(\varphi_{5}+\pi / 2\right) \cdot \omega_{5} \\
& +l_{\mathrm{IM}} \cdot \cos \left(2 \pi-\beta+\varphi_{9}\right) \cdot \omega_{9}
\end{aligned}
$$

The components of the point $\mathrm{M}$ acceleration on both axes are given by the Eq. (3):

$$
\begin{aligned}
a_{x \mathrm{M}}= & -l_{1} \cdot \cos \varphi_{1} \cdot \omega_{1}^{2}-l_{1} \cdot \sin \varphi_{1} \cdot \varepsilon_{1}-l_{\mathrm{BE}} \cdot \cos \varphi_{2} \cdot \omega_{2}^{2} \\
& -l_{\mathrm{BE}} \cdot \sin \varphi_{2} \cdot \varepsilon_{2}-l_{4} \cdot \cos \varphi_{4} \cdot \omega_{4}^{2}-l_{4} \cdot \sin \varphi_{4} \cdot \varepsilon_{4} \\
& -l_{\mathrm{FG}} \cdot \cos \varphi_{5} \cdot \omega_{5}^{2}-l_{\mathrm{FG}} \cdot \sin \varphi_{5} \cdot \omega_{5} \\
& -l_{\mathrm{GI}} \cdot \cos \left(\varphi_{5}+\pi / 2\right) \cdot \omega_{5}^{2}-l_{\mathrm{GI}} \cdot \sin \left(\varphi_{5}+\pi / 2\right) \cdot \varepsilon_{5} \\
& -l_{\mathrm{IM}} \cdot \cos \left(2 \pi-\beta+\varphi_{9}\right) \cdot \omega_{9}^{2} \\
& -l_{\mathrm{IM}} \cdot \sin \left(2 \pi-\beta+\varphi_{9}\right) \cdot \varepsilon_{9} \\
a_{y \mathrm{M}}= & -l_{1} \cdot \sin \varphi_{1} \cdot \omega_{1}^{2}+l_{1} \cdot \cos \varphi_{1} \cdot \varepsilon_{1}-l_{\mathrm{BE}} \cdot \sin \varphi_{2} \cdot \omega_{2}^{2} \\
& +l_{\mathrm{BE}} \cdot \cos \varphi_{2} \cdot \varepsilon_{2}-l_{4} \cdot \sin \varphi_{4} \cdot \omega_{4}^{2}+l_{4} \cdot \cos \varphi_{4} \cdot \varepsilon_{4} \\
& -l_{\mathrm{FG}} \cdot \sin \varphi_{5} \cdot \omega_{5}^{2}+l_{\mathrm{FG}} \cdot \cos \varphi_{5} \cdot \varepsilon_{5} \\
& -l_{\mathrm{GI}} \cdot \sin \left(\varphi_{5}+\pi / 2\right) \cdot \omega_{5}^{2}+l_{\mathrm{GI}} \cdot \cos \left(\varphi_{5}+\pi / 2\right) \cdot \varepsilon_{5} \\
& -l_{\mathrm{IM}} \cdot \sin \left(2 \pi-\beta+\varphi_{9}\right) \cdot \omega_{9}^{2} \\
& +l_{\mathrm{IM}} \cdot \cos \left(2 \pi-\beta+\varphi_{9}\right) \cdot \varepsilon_{9}
\end{aligned}
$$

where: $\varphi_{i}[\mathrm{deg}], i=\overline{2,9}-$ angular variations;

$\omega_{i}[\operatorname{deg} / s], i=\overline{2,9}-$ angular velocity;

$\varepsilon_{i}\left[\mathrm{deg} / s^{2}\right], i=\overline{2,9}-$ angular acceleration. 
Competing interests. The authors declare that they have no conflict of interest.

Edited by: Lotfi Romdhane

Reviewed by: three anonymous referees

\section{References}

Anama, K. and Al-Jumaily, A. A.: Active Exoskeleton Control Systems: State of the Art, Procedia Engineer. 41, 988-994, 2012.

Ashkani, O., Maleki, A., and Jamshidi, N.: Design, simulation and modelling of auxiliary exoskeleton to improve human gait cycle, Australas. Phys. Eng. S., 40, 137-144, https://doi.org/10.1007/s13246-016-0502-6, 2016.

Banala, S. K., Agrawal, S. K., and Scholz, J. P.: Active Leg Exoskeleton (ALEX) for Gait Rehabilitation of Motor-Impaired Patients, Proceedings of the 2007 IEEE 10th International Conference on Rehabilitation Robotics, 12-15 June, Noordwijk, The Netherlands, 401-407, 2007.

Barbareschi, G., Richards, R., Thornton, M., Carlson, T., and Holloway, C.: Statically vs dynamically balanced gait: Analysis of a robotic exoskeleton compared with a human, Conf. Proc. IEEE Eng. Med. Biol. Soc., 2015, 6728-6731, https://doi.org/10.1109/EMBC.2015.7319937, 2015.

Begg, R. K., Wytch, R., and Major, R. E.: Instrumentation used in clinical gait studies: a review, J. Med. Eng. Technol., 13, 290295, 1989.

Bortole, M., Venkatakrishnan, A., Zhu, F., Moreno J. C., Francisco, G. E., Pons J. L., and Contreras-Vidal, J. L.: The H2 robotic exoskeleton for gait rehabilitation after stroke: early findings from a clinical study, J. Neuroeng. Rehabil., 12, PMC4469252, https://doi.org/10.1186/s12984-015-0048, 2015.

Bruzzone, L. and Quaglia, G.: Review article: locomotion systems for ground mobile robots in unstructured environments, Mech. Sci., 3, 49-62, https://doi.org/10.5194/ms-3-49-2012, 2012.

Carbone, G., Ottaviano, E., and Ceccarelli, M.: An optimum design procedure for both serial and parallel manipulators, J. Mech. Eng. Sci., 221, 829-843, 2007.

Carbone, G. and Ceccarelli, M.: A mechanical design of a low-cost easy-operation anthropomorphic wheeled leg for walking machines, Int. J. Robot. Manage., 9, 3-8, 2008.

Ceccarelli, M. and Romdhane, L.: Design issues for humanmachine platform interface in cable-based parallel manipulators for physiotherapy applications, J. Zhejiang Univ. Sci., 11, 231239, https://doi.org/10.1631/jzus.A1000027, 2009.

Ceccarelli, M., Cafolla, D., Wang, M., and Carbone, G.: An Overview of the Ongoing Humanoid Robot Project LARMbot, in: Towards Autonomous Robotic Systems, edited by: Alboul, L., Damian, D., and Aitken, J., TAROS 2016, Lecture Notes in Computer Science, Vol. 9716, Springer, 2016.

Chen, G., Chan, C. K., Guo, Z., and Yu, H.: A review of lower extremity assistive robotic exoskeletons in rehabilitation therapy, Crit. Rev. Biomed. Eng., 41, 343-363, 2013.

Copilusi, C., Ceccarelli, M., and Carbone, G.: Design and numerical characterization of a new leg exoskeleton for motion assistance, Robotica, 33, 1147-1162, 2015.

de Looze, M. P., Bosch, T., Krause, F., Stadler, K. S., O’Sullivan, L. W.: Exoskeletons for industrial application and their poten- tial effects on physical work load, Ergonomics, 59, 671-681, https://doi.org/10.1080/00140139.2015.1081988, 2016.

Díaz, I., Gil, J. J., and Sánchez, E.: Lower-Limb Robotic Rehabilitation: Literature Review and Challenges, J. Robot., 2011, 759764, https://doi.org/10.1155/2011/759764, 2011.

Dollar, A. M. and Herr, H.: Lower extremity exoskeletons and active orthoses: challenges and state-of-the-art, IEEE Trans. Rob., 24, 144-158, 2008.

Dumitru, N., Copilusi, C., Geonea, I., Tarnita, D., and Dumitrache, I.: Dynamic Analysis of an Exoskeleton New Ankle Joint Mechanism, New Trends in Mechanism and Machine Science Mechanisms and Machine Science, Vol. 24, Springer International Publishing, 709-717, 2015.

Geonea, I. D., Alexandru, C., Margine, A., and Ungureanu, A.: Design and simulation of a single DOF humanlike leg mechanism, Appl. Mech. Mat., 332, 491-496, https://doi.org/10.4028/www.scientific.net/AMM.332.491, 2013.

Geonea, I., Ceccarelli, M., and Carbone, G.: Design and analysis of an exoskeleton for people with motor disabilities, in: The 14th IFToMM World Congress, Taipei, Taiwan, 2015.

Hesse, S., Schmidt, H., Werner, C., and Bardeleben, A.: Upper and lower extremity robotic devices for rehabilitation and for studying motor control, Curr. Opin. Neurol., 16, 705-710, 2003.

Ilhem, B., Lepagnot, J., and Siarry, P.: A survey on optimization metaheuristics, Information Sciences, 237, 82-117, https://doi.org/10.1016/j.ins.2013.02.041, 2013.

Jarrassé, N., Proietti, T., Crocher, V., Robertson, J., Sahbani, A., Morel, G., and Roby-Brami, A.: Robotic exoskeletons: a perspective for the rehabilitation of arm coordination in stroke patients, Front. Hum. Neurosci., 1, 8:947, PMC4249450, https://doi.org/10.3389/fnhum.2014.00947, 2014.

Kao, P. C., Lewis, C. L., and Ferris, D. P.: Invariant ankle moment patterns when walking with and without a robotic ankle exoskeleton, J. Biomech., 43, 203-209, 2010.

Kecskemethy, A.: Integrating efficient kinematics in biomechanics of human motions, Procedia IUTAM, 2, 86-92, https://doi.org/10.1016/j.piutam.2011.04.009, 2011.

Lajeunesse, V., Vincent, C., Routhier, F., Careau, E., and Michaud F.: Exoskeletons' design and usefulness evidence according to a systematic review of lower limb exoskeletons used for functional mobility by people with spinal cord injury, Disabil. Rehabil. Assist. Technol., 11, 535-547, https://doi.org/10.3109/17483107.2015, 2016.

Li, T., and Ceccarelli, M.: Design and simulated characteristics of a new biped mechanism, Robotica, 33, 1568-1588, 2015.

Louie, D. R. and Eng, J. J.: Powered robotic exoskeletons in poststroke rehabilitation of gait: a scoping review, J. Neuroeng. Rehabil., 13, 53, https://doi.org/10.1186/s12984-016-0162-5, 2016.

Lu, T. W. and Chang, C. F.: Biomechanics of human movement and its clinical applications, Kaohsiung J. Med. Sci., 28, S1325, 2012.

Meglan, D. A. and Berme, N.: A 3D Passive Mechanical Model of the Human Foot for Use in Locomotion Synthesis, Second North American Congress of Biomechanics, Chicago, 351-352, 1992.

Meng, L., Ceccarelli, M., Yu, Z., Chen, X., and Huang, Q.: An experimental characterization of human falling down, Mech. Sci., 8, 79-89, https://doi.org/10.5194/ms-8-79-2017, 2017. 
Muro-de-la-Herran, A., Garcia-Zapirain, B., and Mendez-Zorrilla, A.: Gait Analysis Methods: An Overview of Wearable and NonWearable Systems, Highlighting Clinical Applications, Sensors, 14, 3362-3394, 2014.

Onose, G., Cârdei, V., Crăciunoiu, Ş. T., Avramescu, V., Opriş, I., Lebedev, M. A., and Constantinescu, M. V.: Mechatronic Wearable Exoskeletons for Bionic Bipedal Standing and Walking: A New Synthetic Approach, Front. Neurosci., 10, 343, https://doi.org/10.3389/fnins.2016.00343, 2016.

Patton, J. L.: Forward Dynamic Modeling of Human Locomotion, Ph.D. Thesis, Michigan State University, 1993.

Perry, J. and Burnfield J. M.: Gait Analysis, Normal and Pathological Function, 2nd Ed, USA, 2010.

Rahman, T., Sample, W., Jayakumar, S., King, M. M., Wee, J. Y., Seliktar, R., Alexander, M., Scavina, M., and Clark, A.: Passive exoskeletons for assisting limb movement, J. Rehabil. Res. Dev., 43, 583-590, 2006.

Rajesh Shah, M.: Design of Human Exo-Skeleton Suit for Rehabilitation of Hemiplegic People, Proc. Eng., 51, 544-553, 2013.

Schorsch, J. F., Keemink, A. Q. L., Stienen, A. H. A., van der Helm, F. C. T., and Abbink, D. A.: A novel self-aligning mechanism to decouple force and torques for a planar exoskeleton joint, Mech. Sci., 5, 29-35, https://doi.org/10.5194/ms-5-29-2014, 2014.

Sutherland, D. H.: The evolution of clinical gait analysis, Part II kinematics, Gait Posture, 16, 159-179, 2002.

Tao, W., Liu, T., Zheng, R., and Feng, H.: Gait analysis using wearable sensors, Sensors, 12, 2255-2283, 2012.

Tarnita, D., Catana, M., and Tarnita, D. N.: Experimental measurement of flexion-extension movement in normal and osteoarthritic human knee, Romanian J. Morphol. Embryol., 54, 309-313, 2013.

Tarnita, D.: Wearable sensors used for human gait analysis, Rom. J. Morphol. Embryol., 57, 373-382, 2016.

Tarnita, D., Geonea, I., Petcu, A., and Tarnita, D. N.: Experimental Characterization of Human Walking on Stairs Applied to $\mathrm{Hu}-$ manoid Dynamics, Advances in Robot Design and Intelligent Control, Springer, 293-301, 2017.

Tedeschi, F. and Carbone, G.: Hexapod Walking Robot Locomotion, In Motion and Operation Planning of Robotic Systems; Springer International Publishing, New York, NY, USA, 439468, 2015.
Tingfang, Y., Cempini, M., Oddoa, C. M., and Vitielloa, N.: Review of assistive strategies in powered lower-limb orthoses and exoskeletons, Robot. Auton. Syst., 64, 120-136, 2015.

Valiant, G. A. (Ed.): Transmission and Attenuation of Heelstrike Accelerations, Human Kinetics Books, Champaign, IL., 1990.

Varela, M. J., Ceccarelli, M., and Flores P.: A kinematic characterization of human walking by using CaTraSys, Mech. Mach. Theory, 86, 125-139, https://doi.org/10.1016/j.mechmachtheory.2014.12.006, 2015.

Veneman, J. F, Kruidhof, R, Hekman, E. E., Ekkelenkamp, R., Van Asseldonk, E. H., and van der Kooij, H.: Design and evaluation of the LOPES exoskeleton robot for interactive gait rehabilitation, IEEE T. Neur. Sys. Reh., 15, 379-386, 2007.

Viteckova, S., Kutilek, P., and Jirina, M.: Wearable lower limb robotics: A review, Journal Biocybern. Biomed. Eng., 33, 96105, https://doi.org/10.1016/j.bbe.2013.03.005, 2013.

Wang, S. and Wang, L.: Design and control of the MINDWALKER exoskeleton, IEEE Trans. Neural. Syst. Reh., 23, 277-286, https://doi.org/10.1109/TNSRE.2014.2365697, 2015a.

Wang, M. F., Ceccarelli, M., and Carbone G.: Experimental tests on operation performance of a LARM leg mechanism with 3-DOF parallel architecture, Mech. Sci., 6, 1-8, https://doi.org/10.5194/ms-6-1-2015, 2015 b.

Winter, D. A.: Biomechanics of Human Movement, John Wiley \& Sons Inc., 1979.

Wojtyra, M.: Multibody Simulation Model of Human Walking, Mechanics Based Design of Structures and Machines, 31, 357-379, 2003.

Young Aaron, J. and Ferris, D. P.: State-of-the-art and Future Directions for Lower Limb Robotic Exoskeletons, IEEE Trans. Neural. Syst. Rehabil. Eng., 171-182, https://doi.org/10.1109/TNSRE.2016.2521160, 2017.

Zoss, A., Kazerooni, H., and Chu, A.: On the mechanical design of the Berkeley Lower Extremity Exoskeleton (BLEEX), IEEE/RSJ International Conference on Intelligent Robots and Systems, 3465-3472, https://doi.org/10.1109/IROS.2005.1545453, 2005. 